BMJ Open Sport \& Exercise Medicine

\title{
SCAT2 and SCAT3 scores at baseline and after sports-related mild brain injury/concussion: qualitative synthesis with weighted means
}

\author{
Roger E Thomas, ${ }^{1}$ Jorge Alves, ${ }^{2}$ Marcus M Vaska, ${ }^{3}$ Rosana Magalhães ${ }^{4}$
}

To cite: Thomas RE, Alves J, Vaska MM, et al. SCAT2 and SCAT3 scores at baseline and after sports-related mild brain injury/concussion: qualitative synthesis with weighted means. BMJ Open Sport Exerc Med 2016;2:e000095. doi:10.1136/bmjsem-2015000095

- Prepublication history and additional material is available. To view please visit the journal (http://dx.doi.org/ 10.1136/bmjsem-2015000095).

Accepted 10 August 2016

CrossMark

\begin{abstract}
${ }^{1}$ Department of Family Medicine, Faculty of Medicine, Health Sciences Centre, University of Calgary, Calgary, Alberta, Canada ${ }^{2}$ CEREBRO-Brain Health Center, Braga, Portugal ${ }^{3}$ Knowledge Resource Service, Alberta Health Services, Holy Cross Centre, Calgary, Alberta, Canada ${ }^{4}$ Clinical Academic CenterBraga, Braga, Portugal
\end{abstract}

Correspondence to Dr Roger E Thomas; rthomas@ucalgary.ca

\section{ABSTRACT}

Objective: Identify all Sport Concussion Assessment Tool (SCAT2/3) studies, compare baseline and postconcussion results.

Design: Systematic review (qualitative synthesis, weighted means).

Data sources: 18 databases, 9 grey literature resources searched for SCAT2/3 data; 9150 articles identified, titles/abstracts assessed/data-entry independently by two reviewers.

Eligibility criteria for selecting studies: Any studies reporting partial/complete SCAT2/3 data.

Results: 21 studies with data (partial/complete data 16 SCAT2 (4087 athletes); 5 SCAT3 (891).

Newcastle-Ottawa risk-of-bias scale: studies with maximum possible score of $4,85 \%$ scored 3 or 4 ; studies with maximum possible score of $6,75 \%$ scored 5 or 6 . SCAT2 high schoolers: weighted mean score for symptoms 18.46 (22=no symptoms), Balance Error Scoring System (BESS) 26.14, Standardised Assessment of Concussion (SAC) 26.00 and SCAT2 total 88.63. Collegiate/ adults weighted means: symptoms 20.09, BESS 25.54, SAC 27.51 and total SCAT2 91.20. Betweenstudy and within-study variability similar to those of the high schoolers. Limited variability between genders. Only 2 studies report baseline and postconcussion scores and 9 partial scores, but data are too limited to provide weighted average scores.

Conclusions: Group mean baseline SCAT scores for high school and collegiate athletes are similar, with minimal gender differences; baseline symptoms show more variability than other components. There are minimal data for elementary students and professionals, no data for adult non-collegiate athletes. Two studies provide preconcussion and postconcussion scores. No data on minimal significant clinical differences to guide players/ coaches in withdrawing from a game in progress and deciding when recovery is complete and play can be resumed. The SCAT needs supplementing with clinical and neuropsychological return-to-play assessments.

\section{What is already known?}

- The Sport Concussion Assessment Tool (SCAT) is the most widely concussion assessment test, but before this review no summary data were available about SCAT component scores or betweensample and within-sample heterogeneity.

\section{What are the new findings?}

21 studies provided SCAT 2/3 assessments and included 4978 athletes (1067 females, 3831 males and 80 gender not stated).

- Only one study reported SCAT data for a professional league (Finnish ice hockey players), there are minimal data on elementary students and no data on adult non-collegiate athletes.

- Each player of a contact sport should complete the SCAT preseason to establish a baseline score.

- The studies with data on preconcussion and postconcussion SCAT scores are too limited to provide weighted average scores. A study of rugby players during 62 games noted 52 concussions (44/1000 match hours) with 8 witnessed concussions, but 44 unwitnessed concussions identified postmatch by the King-Devick test.

\section{INTRODUCTION}

\section{Effects of concussion}

Concussion is typically conceptualised within the range of mild brain injury and may acutely affect cognitive functioning with amnesia, confusion, changes in attention, concentration, memory, information processing, learning and reaction time; cause headaches, dizziness, nausea, vomiting and affect vision, sleep and emotions. ${ }^{1-8}$

The American Medical Society for Sports Medicine $^{9}$ estimated 3.8 million concussions 
occur in the USA annually, and 50\% may be unreported. The rates of concussion per 1000 athlete exposures ranged from 0.17 to 0.23 in two reviews. ${ }^{9} 10$

\section{Follow-up}

The problems experienced by medical teams in follow-up are the often low percentages contactable several months after concussion, ${ }^{11-16}$ and thus we do not know the percentages of individuals who remain with consequences of concussion.

\section{Measurement}

In choosing a concussion measurement tool, it is important that it has excellent validity and reliability and measures concussion in a wide range of sports, age groups and both genders and be equally reliable at initial enquiry and follow-up.

It is important to assess all concussions promptly and prevent individuals returning to contact sports while concussed and especially important to assess subsequent concussions because a history of concussion is associated with a higher risk of subsequent concussions. ${ }^{9}{ }^{17}$ Repeated concussions result in permanent structural changes/ damage and lead to later brain disease. ${ }^{18} 19$

\section{SCAT2/SCAT3}

Several measures have been used to assess concussion: the Postconcussion Scale, the Sport Concussion Assessment Tool (SCAT), the Reporting Information Online, the Concussion Resolution Index and the Graded Symptom Checklist. $^{20}$ The International Symposia on Concussion in Sport Zurich Consensus statement proposed the SCAT2 in 2009. ${ }^{21}$ The SCAT2 includes (1) a 22-item symptom checklist (score of 22=no symptoms), (2) a 2-item physical signs score (loss of consciousness, balance difficulties), a score of $2=$ no problems, (3) the Glasgow Coma Scale (no symptoms= score of 15), (4) the Maddocks Score (five questions about how oriented the player is to the current situation, and the score is not included in the overall SCAT2 score), (5) the Standardised Assessment of Concussion (SAC) (no symptoms=score of 30), (6) the modified Balance Error Scoring System (BESS) (score of 30=no errors), (7) a Coordination examination of five fingernose movements (maximum score 1) and (8) a delayed recall of the component of the SAC which asks for repetition of six words (maximum score 5). The maximum SCAT2 score is 100 . Each symptom can be rated 0-6 to produce a maximum symptom severity score of 132 , which is not considered for the SCAT2 total score.

The 2012 Concussion in Sport Group (CISG) Consensus meeting concluded that there is good evidence to support the use of each of the components scored independently (symptoms, symptom severity, neurocognitive function and balance) but no evidence to support the use of a total score. ${ }^{22-28}$ The individual major components of the SCAT (Symptoms, Balance, SAC) have good validity and reliability. Owing to its wide relevance to many sports and ongoing development, the SCAT is likely to be the key concussion assessment tool and for these reasons is the focus of this review.

\section{Purpose of this systematic review}

Identify all Sport Concussion Assessment Tool (SCAT2/ 3) studies and compare baseline and postconcussion results.

\section{METHODS}

\section{Search strategy}

The following databases were searched using predetermined search strategies discussed between the librarian, principal investigator and coinvestigators: MEDLINE, EMBASE, EBM Reviews (Cochrane Database of Systematic Reviews, ACP Journal Club, Database of Abstracts of Reviews of Effects, Cochrane Central Register of Controlled Trials, Cochrane Methodology Register, Health Technology Assessment, NHS Economic Evaluation Database), PubMed, PubMed Central, Web of Science, CINAHL, SPORTDiscus, Family \& Society Studies Worldwide, Family Studies Abstracts and Health Source-Nursing/Academic Edition. In addition, nine grey literature resources were consulted, including Google, Google Scholar, OpenDOAR (http://www. opendoar.org), Health Sciences Online (HSO) (http:// www.hso.info), ProQuest Dissertations \& Theses, OAISter (http://oaister.worldcat.org), Sports Concussion Institute (http://concussiontreatment.com), Brain Trauma Foundation (https://www.braintrauma.org) and the Canadian Concussion Collaborative (http://casem-acmse. org/education/ccc). No limits on publication date were applied, and the search included studies in all languages and from all countries. All included studies were individually entered in the PubMed single citation matcher, and all relevant citation chains followed up to identify any relevant references.

\section{Eligibility criteria}

On the basis of the information in the abstract, studies had to provide partial or complete SCAT 2 or 3 data and 73 studies were read in full-text.

\section{Data extraction and analysis}

All study titles and abstracts were read independently and data extracted independently by two reviewers (RET, then either JA or RM) and included if the authors reported partial or complete SCAT2 or SCAT3 numerical outcomes.

\section{Data analysis}

For the high school and collegiate age groups and both genders weighted means (according to sample size) were computed for SCAT2 and its components. There were insufficient data for separate computations for elementary school children, ${ }^{29}$ professional athletes ${ }^{30}$ and SCAT 3 scores (table 3); and preconcussion and 
postconcussion scores were compared only for the two studies that provided them. Only one study included data for elementary students (age 9-10 years old) ${ }^{29}$ (table 2), one study provided data for players in a professional league $^{30}$ (table 3) and the SCAT3 studies are too few to permit computation of weighted means; all these data are presented in the tables without further analysis.

\section{Risk of bias assessment}

To assess the overall risk of bias in the selected studies and permit stratification of studies by risk of bias, assessment was performed using the Newcastle-Ottawa Scale (NOS), ${ }^{31}$ developed to assess the quality of nonrandomised studies in meta-analysis. Each study was assessed by two reviewers (RET then either JE or RM) with disagreements resolved by rounds of discussions. Each study was assessed for three components: the selection of the study groups; the comparability of the groups and the ascertainment of either the exposure or outcome of interest for case-control or cohort studies.

\section{RESULTS}

\section{Search}

A total of 9150 articles were identified in all indicated databases and 77 from grey literature and a PubMed search in February 2016. After removal of duplicates, 1267 titles and abstracts which described assessments of concussion using the SCAT 2 and SCAT 3 were identified, 73 papers read in full-text and 21 studies included in this review (figure 1). Among the 21 included studies, 16 reported partial or complete SCAT2 data for 4087 athletes and 16 reported complete or partial SCAT 3 data for 891 athletes (tables 1 and 2, see online supplementary table $\mathrm{S} 1$ ).

\section{Risk of bias}

No studies with interventions were identified and thus the columns for a hemi-cohort exposed to an intervention and the control hemi-cohort in the $\operatorname{NOS}^{31}$ are not filled (table 3). For studies with a maximum possible score of $4,85 \%$ scored 3 or 4 ; and for those with a maximum possible score of $6,75 \%$ scored 5 or 6 .
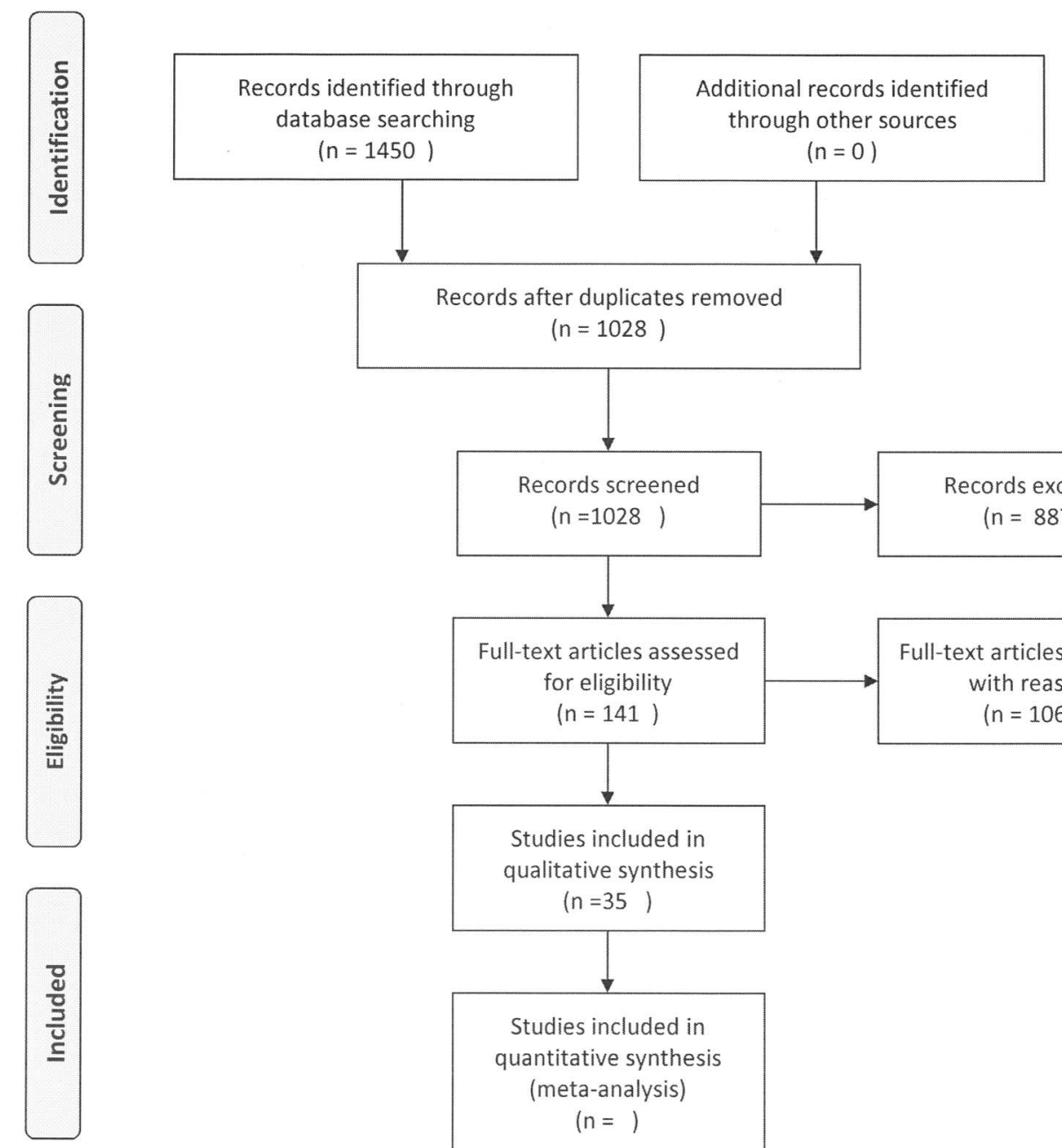

Records after duplicates removed $(n=1028)$

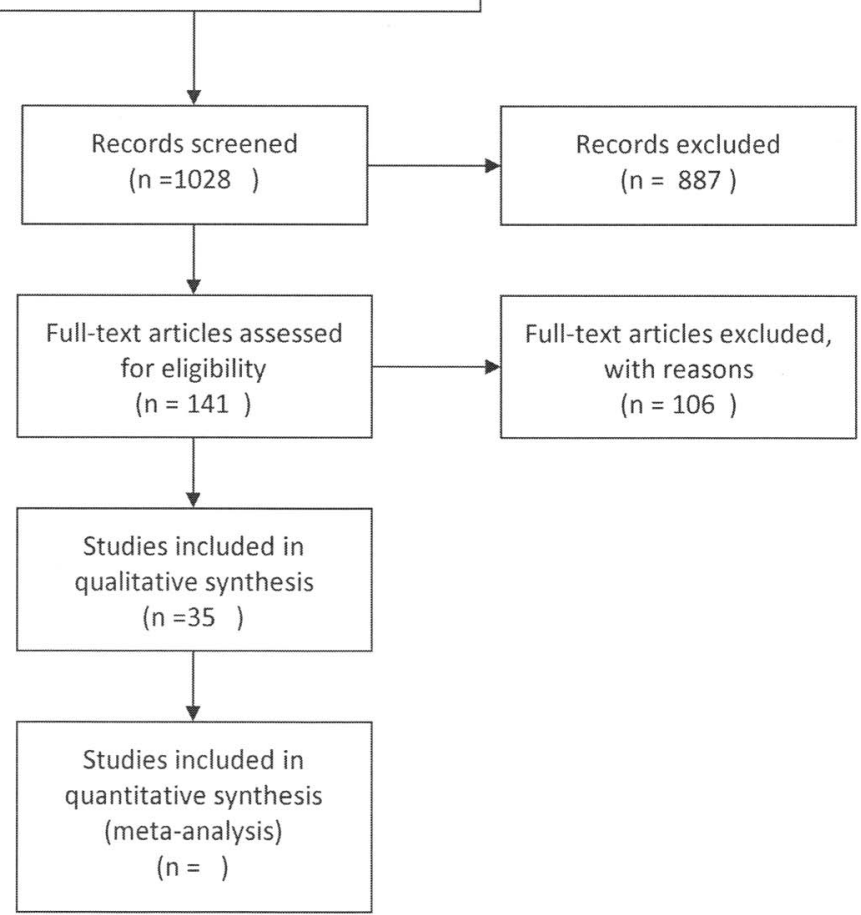

Figure 1 PRISMA 2009 flow diagram. SCAT2 and SCAT3 scores at baseline and after mild brain injury/concussion: systematic review. SCAT, Sport Concussion Assessment Tool. 
\begin{tabular}{ll}
\hline \multicolumn{3}{c}{ Baseline scores } & \\
\cline { 2 - 3 } Author & Symptom score (SD) (perfect \\
score=no symptoms $=22$ points);
\end{tabular}

and date, Participants
country symptom severity score (max=132)

SCAT 2 scores: high schoolers

Echlin, $\quad 67$ male junior male hockey players $18.2(1.2)$ years);

2010, $\quad 21$ concussions medically observed during 51 games

Canada

Glaviano, $\quad(\mathrm{n}=361 ; 195 \mathrm{~m}, 166 \mathrm{f})$; private school, 9 different

2015, USA sports; grades 7-12; avg age 14.6 years

Symptom score avg 20.32

$\begin{array}{ll}\text { Males 20.6 } & \text { Females } 20 \\ \text { (2.0); symptom } & (2.2) ; \\ \text { severity males: } & \text { symptom severity } \\ 19.8(3.9) & \text { females: } 19.1 \\ & (3.8)\end{array}$

Jinguji, $\quad(n=214 ; 155 m, 59 f)$; Seattle high school athletes and $19.75(3.28)$

2012* NW soccer camps

Mayfield, $\quad(n=119 ; 102 m, 15 f, 2$ gender missing); age 15.8 (1.2) $16.4(5.4)$

2013, USA years

(Subset of High school athletes participating in interscholastic

Valovich contact sports

McLeod

2012)

Snyder, $\quad(n=761 ; 656 m, 105 f) ; 9-18$ years. Students attended

2014, USA medical offices for qualifying physical exams

Ages $9-10=18.59(4.5)$ Ages $11-12=19.09(3.77)$ Ages $13-14=19.85(3.17)$

Ages $15-16=20.28(2.73)$

Ages 17-18=20.42 (3.06)

Weighted average ages 9

$18=19.85$

$16.9(5.3)$

18.46

males: 19.1

$25.82(3.45)$

McLeod,

( $n=1134 ; 872 m, 262 f) ;$ high school athletes

USAT

Weighted $2589(1980 \mathrm{~m}, 607 \mathrm{f}) \S$

averagesł

SCAT 2 scores: collegiate/university/adult non-collegiate

Galetta, $\quad(n=27)$ male professional ice hockey team, average

2013, USA age $28 \pm 5$

Mrazik, $\quad(n=125 ; 84 \mathrm{~m}, 41 \mathrm{f})$

2013, undergraduates (95 collegiate athletes and 30

Canada recreational athletes) (14 had concussion history, all

(SCAT2) more than 60 days previously)
22; severity score $=0$

Avg 17.81;

Sport group:

Team sport baseline: $18.6(4.3)$

Individual sport: 16.7 (5.5)

After Leger test: Team 13.5 (8.1),

individual 7.6 (10.3)

Fitness level: baseline

High 19.2 (4.2), low 14.9 (5.2); After

Leger test: high 12.9 (9.0), low 6.3

(9.4)
BESS (Balance) total (SD) (perfect

Avg 26.88

Males 26.6 (2.6) Females 27.2 (2.3)

$27.0(3.4)$

Ages 9-10=23.73 (3.57)

Ages $11-12=25.03(3.43)$

Ages 13-14=24.86 (3.4)

Ages $15-16=25.28(3.58)$

Ages 17-18=25.4 (3.48)

Weighted average ages $9-18=24.91$

26.7 (2.9)

SAC total (SD) (perfect score $=30$ points)

SCAT2 scores (SD) (perfect score $=100$ )

25.75

Avg 26.73;

12-year-olds had the lowes

$\%$ of correct responses for

5 digit (46\%), 6 digit (21\%)

and months backwards

$(67 \%)$ tasks

Males $26.6(2.2)$ Females Males $26.9 \quad 92.0(3.7)$

Females 92.2 (3.8) (2.0)

$25.52(3.06)$

88.99 (7.96)

$26.6(2.6)$

86.0

Ages $9-10=24.22(2.61)$ Ages $11-12=25.26(2.48)$ Ages 13-14=24.7 (3.01)

Ages $15-16=25.26(2.83)$ Ages 17-18-25.47 (2.7) Weighted average ages 9 Weighted

26.5 (2.6)

Avg 92.5

26.14

26.00

88.63

28 (range 25-30)

$\mathrm{n} / \mathrm{a}$

$\mathrm{n} / \mathrm{a}$

n/a 


\begin{tabular}{|c|c|c|c|c|c|}
\hline \multirow[b]{2}{*}{$\begin{array}{l}\text { Author } \\
\text { and date, } \\
\text { country }\end{array}$} & \multicolumn{5}{|l|}{ Baseline scores } \\
\hline & \multirow[t]{2}{*}{ Participants } & $\begin{array}{l}\text { Symptom score (SD) (perfect } \\
\text { score=no symptoms=22 points); } \\
\text { symptom severity score } \\
\text { (max=132) }\end{array}$ & \multirow[t]{2}{*}{$\begin{array}{l}\text { BESS (Balance) total (SD) (perfect } \\
\text { score=30 points) }\end{array}$} & \multirow[t]{2}{*}{$\begin{array}{l}\text { SAC total (SD) (perfect } \\
\text { score }=30 \text { points) }\end{array}$} & \multirow[t]{2}{*}{$\begin{array}{l}\text { SCAT2 scores (SD) (perfect } \\
\text { score }=100 \text { ) }\end{array}$} \\
\hline & & $\begin{array}{ll}\text { Baseline males: } & \text { Baseline } \\
18.7(4.1) ; & \text { females: } 16.0 \\
\text { After Leger } & (5.9) ; \\
20 \mathrm{~m} \text { shuttle-run } & \text { After Leger } 20 \mathrm{~m} \\
\text { test: } & \text { shuttle-run test: } \\
12.5(8.1) & 7.5(11.5)\end{array}$ & & & \\
\hline $\begin{array}{l}\text { Putukian, } \\
2015, \text { USA }\end{array}$ & $\begin{array}{l}(\mathrm{n}=263 ; 176 \mathrm{~m}, 87 \mathrm{f}) \text {; of whom } 178 \text { reported no history } \\
\text { of prior concussion; Princeton University athletes; } \\
\text { average age } 20.33 \text { years }\end{array}$ & $\begin{array}{l}20.52 \text { (2.44); average symptom } \\
\text { severity: } 1.48\end{array}$ & $26.55(3.21)$ & $28.05(1.60)$ & $\begin{array}{l}76.11(4.85) \text { (maximum score }=83 \\
\text { because Glasgow Coma Scale and } \\
\text { physical signs were not measured) }\end{array}$ \\
\hline $\begin{array}{l}\text { Weber, } \\
2013 \text {, USA }\end{array}$ & $\begin{array}{l}(\mathrm{n}=32) \text {; average age } 20.0(1.4) \text { years; Division I male } \\
\text { collegiate wrestlers; tested at: (1) baseline (hydrated); } \\
\text { (2) postpractice (dehydrated) }\end{array}$ & $\begin{array}{l}\text { Symptoms baseline: } 21.56(1.24) \text {; } \\
\text { postpractice: } 16.97(5.23) \\
\text { Symptom severity baseline: } 20.97 \\
\text { (2.91); postpractice: } 9.31(13.80)\end{array}$ & $\begin{array}{l}\text { Baseline: } 15.72 \text { (5.09); postpractice; } 18.81 \\
(6.68)\end{array}$ & $\begin{array}{l}\text { Baseline: } 27.72(1.91) \\
\text { postpractice: } 27.62(2.00)\end{array}$ & $\begin{array}{l}\text { Baseline: } 93.06(3.88) \\
\text { postpractice: } 87.94(6.39)\end{array}$ \\
\hline $\begin{array}{l}\text { Zimmer, } \\
2015 \text { USA }\end{array}$ & $\begin{array}{l}(\mathrm{n}=477 ; 332 \mathrm{~m}, 145 \mathrm{f}) ; 18-23 \text { years. A US National } \\
\text { Collegiate Athletic Association Division }\end{array}$ & $\begin{array}{l}20.25 \text { (3.00); average } 1.75 \\
\text { symptoms }\end{array}$ & $25.64(4.07)$ & $27.17(2.01)$ & $91.08(5.60)$ \\
\hline $\begin{array}{l}\text { Weighted } \\
\text { averages } \ddagger \\
\text { SCAT } 2 \text { sco }\end{array}$ & $\begin{array}{l}\mathrm{n}=924(651 \mathrm{~m}, 273 \mathrm{f}) \\
\text { ores: mixed age samples }\end{array}$ & 20.09 & 25.54 & 27.51 & 91.20 \\
\hline $\begin{array}{l}\text { Carson, } \\
2014, \\
\text { Canada, } \\
\text { SCAT2ף }\end{array}$ & $\begin{array}{l}\text { ( } \mathrm{n}=159 \text { patients with } 170 \text { sports-related concussions; } \\
105 \mathrm{~m}, 65 \mathrm{f}) \text {; data presented for } 159 \text {; no gender data; } \\
41 \text { elementary, } 95 \text { high school, } 34 \text { college/university } \\
\text { students }\end{array}$ & $\begin{array}{l}\text { Symptom severity scores } 26.68 \\
(22.83)\end{array}$ & $\mathrm{n} / \mathrm{a}$ & $\mathrm{n} / \mathrm{a}$ & $\mathrm{n} / \mathrm{a}$ \\
\hline $\begin{array}{l}\text { King, } \\
\text { 2012, New } \\
\text { Zealand } \\
\text { (SCAT2) }\end{array}$ & $\begin{array}{l}50 \text { amateur male rugby league players } \\
\text { All players }(n=50) \\
19.3(4.0) \text { years } \\
\text { Premiers }(n=25) \\
22.1(3.5) \\
\text { Under } 17 \mathrm{~s}(\mathrm{n}=25) \\
16.4(0.7)\end{array}$ & \multirow[t]{2}{*}{$\begin{array}{l}\text { PCSS (median, range) } \\
\text { All players }(n=50) \text {; median scores: } \\
\text { physical, } 0 \text { (range 0-6); cognitive } 0 \\
\text { (range 0-6); sleep 0 (range -2); } \\
\text { emotional (range 0-5) }\end{array}$} & $\mathrm{n} / \mathrm{a}$ & $\mathrm{n} / \mathrm{a}$ & $\mathrm{n} / \mathrm{a}$ \\
\hline $\begin{array}{l}\text { Total } \\
\text { Total all } \\
\text { ages }\end{array}$ & $\begin{array}{l}\mathrm{n}=209 \\
\mathrm{~N}=3597\end{array}$ & & & & \\
\hline $\begin{array}{l}\text { *Jingui } 201 \\
\text { coordinatio } \\
\text { †Excludes } \\
\text { †Weighted } \\
\text { §Reporting } \\
\text { १Additiona } \\
\text { BESS, Bal } \\
\text { Assessme }\end{array}$ & $\begin{array}{l}12 \text { reported the full data for the SCAT2: in addition } \\
\text { on } 0.90 \text { (max 1.00). } \\
\text { Valovich McLeod as did not measure GCS and } \\
\text { averages computed according to numbers of par } \\
\text { g some or all elements of the SACT2. } \\
\text { al data from author; DOI, day of injury; the maximu } \\
\text { lance Error Scoring System; GCS, Glasgow Come } \\
\text { nt Tool. }\end{array}$ & $\begin{array}{l}\text { n to the above four results, he re } \\
\text { Physical Signs test. } \\
\text { rticipants. } \\
\text { um possible total SCAT2 score=1 } \\
\text { a Scales; PCCS, Postconcussior }\end{array}$ & $\begin{array}{l}\text { points. } \\
\text { ymptom Scale; SAC, Standardised }\end{array}$ & ment of Concussion & AT, Sport Concussion \\
\hline
\end{tabular}


Table 2 SCAT3 scores at baseline

\begin{tabular}{|c|c|c|}
\hline $\begin{array}{l}\text { Author, } \\
\text { date, } \\
\text { country }\end{array}$ & Participants & $\begin{array}{l}\text { score=no syn } \\
\text { points); symp } \\
\text { score (max }=1\end{array}$ \\
\hline \multicolumn{3}{|c|}{ Elementary students } \\
\hline $\begin{array}{l}\text { Jennings, } \\
2015 \text {, USA }\end{array}$ & $\begin{array}{l}(n=71) \text {; males; age } 10.2(1.2) \\
\text { years }(1) \text { contact sport group } \\
=\text { football; baseline }=59 \text {, post- } \\
\text { season }(n=54) \text {; non-contact } \\
\text { sport group = baseball C } \\
\text { baseline } n-28 \text {; post season } \\
(n=13)\end{array}$ & (not reported) \\
\hline
\end{tabular}

\section{Collegiate/adults}

Hänninen, $\quad(n=304)$; males; 25.3 (5.2)

2015,

Finland years, $16-40$ years; $60.1 \%$ $(n=181)$ reported one or more previous concussions; average number of concussions sustained prior to testing: $1.2(1.4)$, range $=0-12$; professional male ice hockey players (total in league $=427$ )

Galetta, $\quad(n=332 ; 270 m, 62 f): 243$ 2015, USA (not reported)
20.5 (2.7) (range 0-21.0)

Symptom severity 2.3

(5.2) (range 0-61.0)

\section{BESS (Balance) total (SD) (perfect score $=30$ points) \\ SAC total (SD) (perfect score $=30$ points)}

\section{Contact group; \\ Contact group:} baseline: 27.39 (2.4); postseason: 27.61 (2.0) Non-contact group: baseline: 27.92 (1.8); postseason 28.31 (1.6)

28.0 (2.5) (range 0-20.0) baseline 24.3 (1.9); postseason 25.5 (3.1) Non-contact group: baseline 24.69 (2.2); postseason 24.08 (2.3)

$27.0(1.7)$ (range 19.0-30.0) (not reported)
26 (range 10-30); youth 26 (range 10-30); collegiate 28 (range 21-30)

BESS, Balance Error Scoring System; SAC, Standardised Assessment of Concussion.

\section{Generalisability of the studies}

The most representative study reported on 304 of all the 427 professional male ice hockey league players in Finland (the only study which reported professionals). Five studies focused on one sport: Echlin et $a l^{32}(67$ junior hockey players), King $e t a l^{33}$ (37 rugby union players), King et $a l^{34}$ (104 rugby union and rugby league players), King $e t a l^{35}$ (50 rugby league) and Weber $e t a l^{36}$ (32 wrestlers). Two studies combined reports on two sports: Jennings et $a \ell^{37}$ (71 footballers and baseball players) and Galetta $e t a l^{38}$ (332 ice hockey and lacrosse players). Four studies reported individuals seen in concussion clinics. ${ }^{39-42}$ The rest reported participants in multiple sports with up to nine different sports. ${ }^{43}$

\section{Variability within and between studies}

Weighted mean SCAT 2 scores were similar for the high school and collegiate/university/adult non-university athletes (table 4).

Symptom scores showed more variability between samples than the other SCAT2 components. For high school children, the lowest mean SCAT symptom scores $\left(22=\right.$ no symptoms) were $12.5\left(\right.$ Baker $\left.^{40}\right) 16.4$ (Mayfield) ${ }^{48}$ and 16.9 (Valovich McLeod) ${ }^{27}$ with the SDs ranging from 2 to 6.7. The lowest mean BESS score was 23.73 (Snyder and Bauer ${ }^{29}$ for those aged 9-10); SDs ranged from 2.0 to 3.58. The lowest mean SAC score was $24.22^{29}$ for those aged 9 and 10; SDs ranged from 1.9 to 3.01 . The lowest mean SCAT2 total score was $84.35 ;{ }^{29}$ SDs ranged from 3.7 to 7.55 (Snyder and Bauer ${ }^{29}$ for those aged 9 and 10), whereas in some studies (eg, Zimmer et $a l^{44}$ ) there was minimal variability. For collegiate/adult athletes between-study and within-study variability were similar to those of the high school children. For the two SCAT3 studies that provided complete data, Finnish ice hockey players ${ }^{30}$ had symptom scores of 20.5, BESS of 28.0 and SAC of 27.0 and for Benedict $e t a l^{39} 14,24.1$, and 27.

\section{Variability in SAC scores}

Three studies identified inaccuracy in repetition tasks that might account for variability in SAC scores. Among 13-15-year-old boys, $69 \%$ could repeat 4 digits correctly, $37 \% 5$ digits, $12 \% 6$ digits and $57 \%$ could say the months of the year backwards. ${ }^{45}$ Among 16-19-year-old males $79 \%$ could repeat 4 digits correctly, $37 \% 6$ digits, $21 \% 6$ digits and $70 \%$ could say the months of the year backwards. ${ }^{45}$ Among 12-year-old boys $85 \%$ repeated 4 digits correctly, $65 \% 5$ digits, $31 \% 6$ digits and $65 \%$ could say the months of the year backwards. ${ }^{43}$ Among 18-year-old men $100 \%$ repeated 4 digits correctly, $82 \% 5$ digits, $72 \% 6$ digits and $82 \%$ could say the months of the year backwards. ${ }^{43}$ Among college athletes (average age 20, range 17-32) SAC scores were higher than for 
Table 3 Assessments of risk of bias in included studies, according to the NOS

Selection

Comparability

Outcome

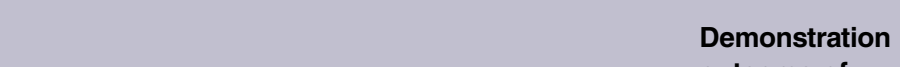

Representativeness Selection of

Author, date

of exposed

cohortst

cohort‡

outcome of

interest not

Studies providing complete preconcussion or preconcussion and postconcussion data

Benedict, 2015

Glaviano, 2015

Hänninen, 2015

Jinguji, 2012

Mayfield, 2013

Putukian, 2015

Snyder, 2014

Valovich McLeod,

2012

Weber, 2013

Zimmer, 2015

Studies presenting partial data

Baker, 2015

Carson, 2014

Echlin, 2010

Galetta, 2013

Galetta, 2015

Jennings, 2015

King, 2012

King, 2013

King, 2015

Miller, 2015

Mrazik, 2013

0
0
$*$
0
$*$
0
$*$
$*$
$*$
$*$


0
0
0
0
0
$*$
$*$
0
0
0
0
0

remparability

Outcome

\begin{tabular}{|c|c|c|c|}
\hline $\begin{array}{l}\text { Follow-up } \\
\text { long } \\
\text { enough } \\
\text { for } \\
\text { outcomes } \\
\text { to occur** }\end{array}$ & $\begin{array}{l}\text { Adequacy } \\
\text { of } \\
\text { follow-up } \\
\text { of } \\
\text { cohorts }\end{array}$ & $\begin{array}{l}\text { Potential } \\
\text { maximum } \\
\text { score }\end{array}$ & $\begin{array}{l}\text { Actual } \\
\text { score }\end{array}$ \\
\hline
\end{tabular}

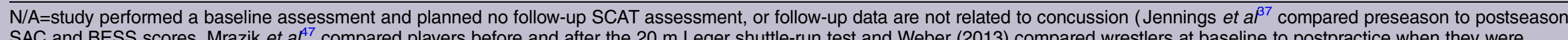
SAC and BESS scores, Mrazik et $a f^{47}$ compared players before and after the $20 \mathrm{~m}$ Leger shuttle-run test and Weber (2013) compared wrestlers at baseline to postpractice when they were dehydrated).

†ls the exposed (preteenaged to high school population with active sports participation assessed with SCAT2 or SCAT3 tools) cohort truly or somewhat representative of young athletes who suffer sports-related concussions/are exposed to sports-related concussions?

łBecause this review identified no studies with interventions, none included a hemi-cohort exposed to an intervention and a hemi-cohort which was the control.

§Exposure is defined as a competitive athlete who has received a complete or partial SCAT2 or SCAT3 assessment.

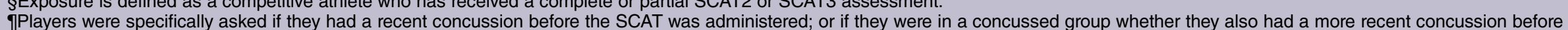
testing.

${ }_{* \star}$ Single asterisk=complete follow-up, OR if participants lost to follow-up were unlikely to introduce bias (according to NOS instructions).

BESS, Balance Error Scoring System; NOS, Newcastle-Ottawa Scale; SAC, Standardised Assessment of Concussion; SCAT, Sport Concussion Assessment Tool; SCAT, Sport Concussion

Assessment Tool. 
Table 4 Weighted mean SCAT2 scores by the age group

\begin{tabular}{lllll}
\hline & $\begin{array}{l}\text { Symptom score (SD) } \\
\text { (perfect score=no }\end{array}$ & $\begin{array}{l}\text { BESS (Balance) total } \\
\text { (SD) (perfect } \\
\text { symptoms=22 points) }\end{array}$ & $\begin{array}{l}\text { SAC total (SD) } \\
\text { (perfect score=30 }\end{array}$ & $\begin{array}{l}\text { SCAT2 scores (SD) } \\
\text { (perfect }\end{array}$ \\
scoup points) & points) & 88.63 \\
\hline High school students & 18.46 & 26.14 & 26.00 & 91.20 \\
Collegiate/university/ & 20.09 & 25.54 & 27.51 & \\
adult non-university & & & & \\
\hline BESS, Balance Error Scoring System; SAC, Standardised Assessment of Concussion; SCAT, Sport Concussion Assessment Tool.
\end{tabular}

high school students (eg, 51\% for 6 digits and 92\% months of the year). ${ }^{46}$ Female data were similar. ${ }^{46}$

\section{Variability in scores before and after tasks without concussion}

One study reported differences in symptoms after exercise (females symptom score 16.0 pre-exercise, females symptom score 11.5 after Leger 20 metre shuttle run test. Males symptoms score 18.7 pre-exercise, males symptom score 12.5 postexercise) ${ }^{47}$ while another study did not (total symptoms (21.56, 20.97), BESS (15.72, 18.81), SAC (27.72, 27.62) and SCAT2 total (93.06, 87.94)). ${ }^{36}$

\section{Rates of detection of concussion}

King et $a l^{33}$ found that during amateur rugby union matches five concussions were identified by a nurse at the pitch (average SCAT2 60.6, SD 4.2) but 17 were identified later by the King-Devick test with much higher SCAT2 total scores: (total SCAT2 84.2, SD 9.6). King $e t a l^{34}$ during rugby union and league games noted eight witnessed concussions: total symptoms (8.6, SD 3.7), BESS (17.5, SD 3.4), SAC (23.0, SD 2.1) and 44 unwitnessed concussions with a difference only in those with lower BESS scores: total symptoms (8.6, SD 4.8), BESS (12.5, SD 6.2) and SAC 23.5, SD 3.1).

\section{Studies of concussed players}

Only two studies report baseline and postconcussion scores (see online supplementary table S1). Mayfield et $a l^{48}$ reported data from the day of concussion to the 10th day after concussion for 119 concussed athletes and found most improvement in symptom scores: baseline total symptoms were 16.54 (day of impact 10.9; day 314 . 8; day 10 19.9); BESS baseline 27.0 (day of impact 24.5; day 326.0 ; day 1027.4 ); SAC baseline 26.6 (day of impact 25.2; day 326.3 ; day 10 26.6) and total SCAT2 baseline 88.00 (day of impact 77.76; day 384.74 ; day 10 91.68). Putukian et $a l^{49}$ for 32 concussed players found their baseline Total Symptom Score was 19.44 and postconcussion 13.00, BESS 26.13 and 24.54, SAC 27.63 and 27.13 and SCAT2 total (excluding the Glasgow Coma Scale and physical signs) 74.16 and 65.28. The weighted averages for these two studies combined show an increase in postconcussion of 8 symptoms, a decrease of 2.3 on the BESS and an increase of 1 on the SAC. Nine studies report partial data for concussed players (see online supplementary table S1), and the incomplete preconcussion and postconcussion data are insufficient to provide reliable weighted average SCAT scores.

\section{Gender differences}

There were sufficient gender data to compute weighted mean SCAT2 scores only for high school students and these showed minimal differences between genders. For females weighted mean symptom scores were 18.44 (males 18.86), BESS scores 26.75 (males 25.89), SAC scores 26.76 (males 26.03), and total SCAT 2 scores 90.10 (males 88.79) (table 5).

\section{DISCUSSION}

\section{Amount of published SCAT2/3 data}

This systematic review includes SCAT data from 4978 athletes. Most studies $(\mathrm{n}=16)$ provided partial or complete SCAT2 data, and five provided partial or complete SCAT3 data. There are limited data for elementary students, data only for one professional league and none for adult non-collegiate athletes. Generalisability was highest for a study of Finnish professional male ice hockey which assessed most players in the league ${ }^{30}$ and lowest for studies of individual schools or groups including many sports. SCAT component average scores were homogeneous between samples, but there was considerable heterogeneity within samples. Available studies suggest no strong differences between genders.

\section{Limited data postconcussion}

There are limited data on concussion. Only two studies $(\mathrm{n}=151)$ provided preconcussion and postconcussion data. A study of 104 rugby players during 62 games noted 52 concussions (44/1000 match hours) with 8 witnessed concussions, but 44 unwitnessed concussions which were identified postmatch by the King-Devick test. $^{34}$

\section{Preseason baseline SCAT scores}

Group averages are not perfect scores on all SCAT components preseason because some athletes had preexisting symptoms (eg, headache) or because some athletes had problems with test items (eg, repeating serial numbers backwards). Thus, it is important to test players at least preseason and also during the season to detect unwitnessed concussions. 
Table 5 SCAT2 scores: gender differences

\begin{tabular}{|c|c|c|c|c|c|}
\hline \multirow[b]{2}{*}{$\begin{array}{l}\text { Author, date, country, and } \mathrm{N} \\
\text { participants }\end{array}$} & \multicolumn{5}{|l|}{ Baseline scores } \\
\hline & Symptom score (SD) & BESS (SD) & SAC (SD) & SCAT2 (SD) & $\begin{array}{l}\text { Symptom } \\
\text { severity }\end{array}$ \\
\hline \multicolumn{6}{|l|}{ High schoolers } \\
\hline $\begin{array}{l}\text { Glaviano, 2015, USA, females } \\
(n=166)\end{array}$ & $\begin{array}{l}20(2.2) \text {; symptom } \\
\text { severity } 19.1(3.8)\end{array}$ & $27.2(2.3)$ & $\begin{array}{l}26.9(2.0): 12 \text {-year-olds had the lowest \% of correct responses for } \\
4 \text { digit }(88 \%), 5 \text { digit }(27 \%), 6 \text { digit }(12 \%) \text { and months backwards } \\
(69 \%) \text { tasks compared to } 18 \text {-year-old females } 4 \text { digit }(100 \%), 5 \\
\text { digit }(60 \%), 6 \text { digit }(20 \%) \text { and months backwards }(100 \%)\end{array}$ & $92.2(3.8)$ & $2.9(3.8)$ \\
\hline $\begin{array}{l}\text { Glaviano, 2015, USA, males } \\
(n=195)\end{array}$ & $\begin{array}{l}20.6(2.0) ; \text { symptom } \\
\text { severity } 19.8(3.9)\end{array}$ & $26.6(2.6)$ & $\begin{array}{l}26.6(2.2), 12 \text {-year olds had the lowest } \% \text { of correct responses for } \\
4 \text { digit }(85 \%), 5 \text { digit }(65 \%), 6 \text { digit }(31 \%) \text { and months backwards } \\
(65 \%) \text { tasks compared to } 18 \text {-year-old males } 4 \text { digit }(100 \%), 5 \\
\text { digit }(82 \%), 6 \text { digit }(72 \%) \text { and months backwards }(82 \%)\end{array}$ & $92.0(3.7)$ & $2.2(3.9)$ \\
\hline Snyder, 2014, females $(n=105)$ & $18.32(4.69)$ & $25.57(3.45)$ & $25.19(3.04)$ & $86.97^{\star}(6.97)$ & $6.35(9.25)^{\star}$ \\
\hline Snyder, 2014 , males $(n=656)$ & $20.25(2.82)$ & $25.07(3.53)$ & $25.1(2.78)$ & $88.36^{*}(5.61)$ & $2.98(5.79)^{\star}$ \\
\hline $\begin{array}{l}\text { Jinguji, } 2012 \text {, females }(n=59 ; 28 \\
\text { age } 13-15,31 \text { age } 16-19)\end{array}$ & $\begin{array}{l}13-15 \text { years } 20.89 \\
(2.79) ; 16-19 \text { years } \\
18.59(4.74)\end{array}$ & $\begin{array}{l}13-15 \text { years } 27.41 \\
(2.14) ; 16-19 \text { years } \\
26.83(3.00)\end{array}$ & $13-15$ years 26.07 (3.02); $16-19$ years $26.67(2.59)$ & $\begin{array}{l}13-15 \text { years } 92.33 \\
(4.29) ; 16-19 \text { years } \\
89.55(6.47)\end{array}$ & $\mathrm{n} / \mathrm{a}$ \\
\hline $\begin{array}{l}\text { Jinguji, } 2012 \text {, males }(n=155 ; 83 \\
\text { age } 13-15,72 \text { age } 16-19)\end{array}$ & $\begin{array}{l}13-15 \text { years } 20.14 \\
(2.43) ; 16-19 \text { years } \\
19.37(3.38)\end{array}$ & $\begin{array}{l}13-15 \text { years } 25.25 \\
(3.51) ; 16-19 \text { years } \\
25.65(3.78)\end{array}$ & $13-15$ years $25.06(2.50) ; 16-19$ years $25.41(3.69)$ & $\begin{array}{l}13-15 \text { years } 88.37 \\
(5.49) ; 16-19 \text { years } \\
88.28(6.39)\end{array}$ & $\mathrm{n} / \mathrm{a}$ \\
\hline $\begin{array}{l}\text { Valovich McLeod, 2012, females } \\
(n=262)\end{array}$ & $16.3(5.8)$ & $27.3(2.7)$ & $26.9(2.3)$ & $88.7(6.8)^{*}$ & $\mathrm{n} / \mathrm{a}$ \\
\hline $\begin{array}{l}\text { Valovich McLeod, 2012, males } \\
(\mathrm{n}=872)\end{array}$ & $16.7(5.3)$ & $26.6(2.9)$ & $26.4(2.7)$ & $87.7(6.8)^{\star}$ & $\mathrm{n} / \mathrm{a}$ \\
\hline Zimmer, 2015 , females $(n=144)$ & 20.09 (3.29) & $25.94(3.90)$ & $27.63 \dagger(1.87)$ & $91.65(5.58)$ & $\mathrm{n} / \mathrm{a}$ \\
\hline Zimmer, 2015, males $(n=330)$ & $20.31(2.87)$ & $25.49(4.14)$ & $26.97 \dagger(2.05)$ & $90.83(5.60)$ & $\mathrm{n} / \mathrm{a}$ \\
\hline Weighted averages & $\begin{array}{l}\text { Females: } 18.44 \\
\text { Males: } 18.86\end{array}$ & $\begin{array}{l}\text { Females: } 26.75 \\
\text { Males: } 25.89\end{array}$ & $\begin{array}{l}\text { Females: } 26.76 \\
\text { Males: } 26.03\end{array}$ & $\begin{array}{l}\text { Females: } 90.10 \\
\text { Males: } 88.79\end{array}$ & \\
\hline Totals & 2945 (737f, 2208m) & & & & \\
\hline University & & & & & \\
\hline $\begin{array}{l}\text { Mrazik, 2013, Canada, females } \\
(n=41)\end{array}$ & $\begin{array}{l}\text { Baseline: } 6.0 \text { (5.9) } \\
\text { After Leger } 20 \text { metre } \\
\text { shuttle-run test: } 7.5 \\
(11.5)\end{array}$ & $\mathrm{n} / \mathrm{a}$ & $\mathrm{n} / \mathrm{a}$ & $\mathrm{n} / \mathrm{a}$ & $\mathrm{n} / \mathrm{a}$ \\
\hline $\begin{array}{l}\text { Mrazik, 2013, Canada, males } \\
(n=84)\end{array}$ & $\begin{array}{l}\text { Baseline: } 18.7(4.1) \\
\text { After Leger } 20 \text { metre } \\
\text { shuttle-run test: } 12.5 \\
(8.1)\end{array}$ & $\mathrm{n} / \mathrm{a}$ & $\mathrm{n} / \mathrm{a}$ & $\mathrm{n} / \mathrm{a}$ & $\mathrm{n} / \mathrm{a}$ \\
\hline Total all age groups & $3070(778 f, 2292 m)$ & & & & \\
\hline
\end{tabular}




\section{Changes in SCAT scores during practice and play}

There are only two studies which report how SCAT component scores change without a concussion when players exercise or become exhausted during play, and more studies are needed during game intermissions to assess the effects of play and exhaustion so that when a concussion occurs the total change after a concussion can be partitioned into the effects of play and concussion. General postevent testing (eg, King-Devick test) is important in identifying concussions which are missed or unwitnessed during gameplay.

There are insufficient data on the longitudinal normative and abnormal range of SCAT performance in athletes, and this limits its current use as a science-based diagnostic and prognostic tool to guide players and coaches when to stop players continuing during matches and when to allow them to resume their sport.

\section{Strengths and limitations of this review}

We searched 18 databases and 9 grey literature resources without limitation of language or date. We could not combine data from elementary, high school and collegiate players because of the differences in physical size, the multiple sports played and individual sports results not provided and likely differences in competitiveness and force of contacts. Instead, we report for each age group means weighted by the study sample sizes. The effects of heterogeneity within and between samples were thus not explored statistically. Owing to the limited amount of data for many sports and age groups, the absence of data for adult non-collegiate players and data available only for one professional league, we were not able to draw conclusions for these groups.

\section{CONCLUSIONS}

SCAT2/3 data are available for 4978 athletes, but there are minimal data for elementary school students, professional athletes and concussed athletes. There is no uniformity across sports in preseason SCAT testing to obtain baseline scores, numbers of observers to increase the likelihood of detecting concussions during play or using King-Devick tests to detect unwitnessed concussions. There is an urgent need for systematic adoption of continuous follow-up assessments including complete SCAT and King-Devick testing in all age ranges of amateur and professional athletes participating in sports with risk of concussion and follow them if concussed until fully recovered. Minimum clinically important differences in SCAT scores ${ }^{37}$ have not been defined and would need to be based on careful assessments by several assessors with minimal intraobserver and interobserver variability and detailed follow-up of athletes to determine full recovery and appropriate return to play. Until these problems have been solved, the SCAT should be used cautiously for clinical and return-to-play decision processes and be complemented with an extensive medical examination and quantitative neuropsychological testing providing objective performance decrement data.

Acknowledgements The authors thank Dr Benedict and Carson for additional data.

Contributors RET conceived the systematic review, assessed titles and abstracts from the search, entered data into the review, computed data and wrote all drafts of the article. MMV conducted the searches. Titles/abstracts were assessed and data independently entered into the text and tables, and the text was edited and amplified by JA, RM and MMV. All authors agree with the final version of the article.

Competing interests None declared.

Provenance and peer review Not commissioned; externally peer reviewed.

Data sharing statement No additional data are available.

Open Access This is an Open Access article distributed in accordance with the Creative Commons Attribution Non Commercial (CC BY-NC 4.0) license, which permits others to distribute, remix, adapt, build upon this work noncommercially, and license their derivative works on different terms, provided the original work is properly cited and the use is non-commercial. See: http:// creativecommons.org/licenses/by-nc/4.0/

\section{REFERENCES}

1. Collie A, Makdissi M, Maruff $P$, et al. Cognition in the days following concussion: comparison of symptomatic versus asymptomatic athletes. J Neurol Neurosurg Psychiatry 2006;77:241-5.

2. Guskiewicz KM, McCrea M, Marshall SW, et al. Cumulative effects associated with recurrent concussion in collegiate football players: the NCAA Concussion Study. JAMA 2003;290:2549-55.

3. Echemendia RJ, Julian LJ. Mild traumatic brain injury in sports: neuropsychology's contribution to a developing field. Neuropsychol Rev 2001;11:69-88.

4. Erlanger D, Saliba E, Barth J, et al. Monitoring resolution of postconcussion symptoms in athletes: preliminary results of a web-based neuropsychological test protocol. J Athl Train 2001;36:280-7.

5. Macciocchi SN, Barth JT, Alves W, et al. Neuropsychological functioning and recovery after mild head injury in collegiate athletes. Neurosurgery 1996;39:510-4.

6. Pardini D, Stump J, Lovell M, et al. The post-concussion symptom scale (PCSS): a factor analysis. Br J Sports Med 2004;38:661-2.

7. Pellman EJ, Viano DC, Casson IR, et al. Concussion in professional football: injuries involving 7 or more days out-part 5 . Neurosurgery 2004;55:1100-19.

8. Williamson IJ, Goodman D. Converging evidence for the under-reporting of concussions in youth ice hockey. $\mathrm{Br} J$ Sports $\mathrm{Med}$ 2006;40:128-32; discussion 128-32.

9. Harmon KG, Drezner J, Gammons M, et al. American Medical Society for Sports Medicine position statement: concussion in sport. Clin J Sport Med 2013;23:1-18.

10. Pfister T, Pfister K, Hagel B, et al. The incidence of concussion in youth sports: a systematic review and meta-analysis. $\mathrm{Br} J$ Sports Med 2016;50:292-7.

11. Pickering A, Grundy K, Clarke A, et al. A cohort study of outcomes following head injury among children and young adults in full-time education. Emerg Med J 2012;29:451-4.

12. Lowdon IM, Briggs M, Cockin J. Post-concussional symptoms following minor head injury. Injury 1989;20:193-4.

13. Wade DT, Crawford S, Wenden FJ, et al. Does routine follow up after head injury help? A randomised controlled trial. J Neurol Neurosurg Psychiatry 1997;62:478-84.

14. Wade DT, King NS, Wenden FJ, et al. Routine follow up after head injury: a second randomised controlled trial. J Neurol Neurosurg Psychiatry 1998;65:177-83.

15. Elgmark Andersson E, Emanuelson I, Björklund R, et al. Mild traumatic brain injuries: the impact of early intervention on late sequelae. A randomized controlled trial. Acta Neurochir (Wien) 2007;149:151-9; discussion 160.

16. Jakola AS, Müller K, Larsen M, et al. Five-year outcome after mild head injury: a prospective controlled study. Acta Neurol Scand 2007;115:398-402.

17. King D, Brughelli M, Hume $\mathrm{P}$, et al. Assessment, management and knowledge of sport-related concussion: systematic review. Sports Med 2014;44:449-71. 
18. Gavett BE, Stern RA, McKee AC. Chronic traumatic encephalopathy: a potential late effect of sport-related concussive and subconcussive head trauma. Clin Sports Med 2011;30:179-88, xi.

19. Riley DO, Robbins CA, Cantu RC, et al. Chronic traumatic encephalopathy: contributions from the Boston University Center for the Study of Traumatic Encephalopathy. Brain Inj 2015;29:154-63.

20. Brown DA, Elsass JA, Miller AJ, et al. Differences in symptom reporting between males and females at baseline and after a sports-related concussion: a systematic review and meta-analysis. Sports Med 2015;45:1027-40.

21. McCrory $\mathrm{P}$, Meeuwisse $\mathrm{W}$, Johnston $\mathrm{K}$, et al. Consensus statement on concussion in sport-the Third International Conference on Concussion in Sport held in Zurich, November 2008. Phys Sportsmed 2009;37:141-59.

22. Guskiewicz KM, Register-Mihalik J, McCrory P, et al. Evidence-based approach to revising the SCAT2: introducing the SCAT3. Br J Sports Med 2013;47:289-93.

23. McCrea M, Kelly JP, Kluge J, et al. Standardized assessment of concussion in football players. Neurology 1997;48:586-8.

24. McCrea M, Kelly JP, Randolph C, et al. Standardized assessment of concussion (SAC): on-site mental status evaluation of the athlete. $J$ Head Trauma Rehabil 1998;13:27-35.

25. Koscs M, Kaminski TW, Swanik CB, et al. Effects of exertional exercise on the Standardized Assessment of Concussion (SAC) Score. Athl Train Sports Health Care 2009;1:24-30.

26. Barr WB, McCrea M. Sensitivity and specificity of standardized neurocognitive testing immediately following sports concussion. $J$ Int Neuropsychol Soc 2001;7:693-702.

27. Valovich McLeod TC, Bay RC, Lam KC, et al. Representative baseline values on the Sport Concussion Assessment Tool 2 (SCAT2) in adolescent athletes vary by gender, grade, and concussion history. Am J Sports Med 2012;40:927-33.

28. Riemann BL, Guskiewicz KM. Effects of mild head injury on postura stability as measured through clinical balance testing. J Athl Train 2000;35:19-25.

29. Snyder AR, Bauer RM, Health IMPACTS for Florida Network. A normative study of the sport concussion assessment tool (SCAT2) in children and adolescents. Clin Neuropsychol 2014;28:1091-103.

30. Hänninen T, Tuominen M, Parkkari J, et al. Sport concussion assessment tool-3rd edition-normative reference values for professional ice hockey players. J Sci Med Sport 2016;19:636-41.

31. Wells G, Shea B, O'Connell D. The Newcastle-Ottawa Scale (NOS) for assessing the quality of nonrandomised studies in meta-analyses. 2014. http://www.ohri.ca/programs/clinical_ epidemiology/oxford.asp

32. Echlin PS, Tator $\mathrm{CH}$, Cusimano MD, et al. A prospective study of physician-observed concussions during junior ice hockey: implications for incidence rates. Neurosurg Focus 2010;29:E4.

33. King D, Brughelli M, Hume $P$, et al. Concussions in amateur rugby union identified with the use of a rapid visual screening tool. J Neurol Sci 2013;326:59-63.
34. King D, Gissane C, Hume PA, et al. The King-Devick test was useful in management of concussion in amateur rugby union and rugby league in New Zealand. J Neurol Sci 2015;351:58-64.

35. King D, Clark T, Gissane C. Use of a rapid visual screening tool for the assessment of concussion in amateur rugby league: a pilot study. J Neurol Sci 2012;320:16-21.

36. Weber AF, Mihalik JP, Register-Mihalik JK, et al. Dehydration and performance on clinical concussion measures in collegiate wrestlers. J Athl Train 2013;48:153-60.

37. Jennings $D$, Sells $P$, Allison J, et al. Effects of a season of subconcussive contact on Child-Scat3 Scores in 8-12 year-old male athletes. Int J Sports Phys Ther 2015;10:667-75.

38. Galetta KM, Morganroth J, Moehringer N, et al. Adding vision to concussion testing: a prospective study of sideline testing in youth and collegiate athletes. J Neuroophthalmol 2015;35:235-41.

39. Benedict PA, Baner NV, Harrold GK, et al. Gender and age predict outcomes of cognitive, balance and vision testing in a multidisciplinary concussion center. J Neurol Sci 2015;353: 111-5.

40. Baker JG, Leddy JJ, Darling SR, et al. Factors associated with problems for adolescents returning to the classroom after sport-related concussion. Clin Pediatr (Phila) 2015;54:961-8.

41. Darling SR, Leddy JJ, Baker JG, et al. Evaluation of the Zurich Guidelines and exercise testing for return to play in adolescents following concussion. Clin J Sport Med 2014;24:128-33.

42. Miller JH, Gill C, Kuhn EN, et al. Predictors of delayed recovery following pediatric sports-related concussion: a case-control study. J Neurosurg Pediatr 2016;17:491-6.

43. Glaviano NR, Benson S, Goodkin HP, et al. Baseline SCAT2 Assessment of healthy youth student-athletes: preliminary evidence for the use of the child-SCAT3 in children younger than 13 years. Clin J Sport Med 2015;25:373-9.

44. Zimmer A, Marcinak J, Hibyan S, et al. Normative values of major SCAT2 and SCAT3 components for a college athlete population. Appl Neuropsychol Adult 2015;22:132-40.

45. Jinguji TM, Bompadre V, Harmon KG, et al. Sport Concussion Assessment Tool-2: baseline values for high school athletes. Br J Sports Med 2012;46:365-70.

46. Shehata N, Wiley JP, Richea S, et al. Sport concussion assessment tool: baseline values for varsity collision sport athletes. $\mathrm{Br} J$ Sports Med 2009;43:730-4.

47. Mrazik M, Naidu D, Lebrun C, et al. Does an individual's fitness leve affect baseline concussion symptoms? J Athl Train 2013;48:654-8.

48. Mayfield R, Bay R, Valovich McLeod T. Postconcussion deficits measured by the Sport Concussion Assessment Tool 2 among interscholastic athletes. Athl Train Sports Health Care 2013:5:265-71.

49. Putukian M, Echemendia R, Dettwiler-Danspeckgruber A, et al. Prospective clinical assessment using Sideline Concussion Assessment Tool-2 testing in the evaluation of sport-related concussion in college athletes. Clin J Sport Med 2015;25:36-42. 